\title{
Grain Boundaries in Ceramics and Ceramic-Metal Interfaces
}

\author{
D. R. Clarke
}

IBM Research Division

CONF-8608144--1

Yorktown Heights, NY 10598

DE87 001514

\section{Dieter Wolf*}

Materials Science and Technology Division Argonne National Laboratory, Argonne, IL 60439

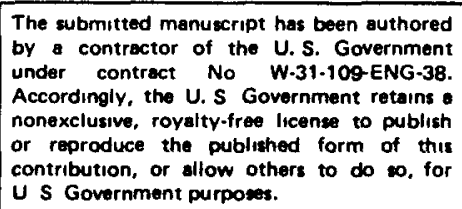

\begin{abstract}
DISCLAIMER
This report was prepared as an account of work sponsored by an agency of the United States Government. Neither the United States Government nor any agency thereof, nor any of their employees, makes any warranty, express or implied, or assumes any legal liability or responsibility for the accuracy, completeness, or usefulness of any information, apparatus, product, or process disclosed, or represents that its use would not infringe privately owned rights. Reference herein to any specific commercial product, process, or service by trade name, trademark, manufacturer, or otherwise does not necessarily constitute or imply its endorsement, recommendation, or favoring by the United States Government or any agency thereof. The views and opinions of authors expressed herein do not necessarily state or reflect those of the United States Government or any agency thereof.
\end{abstract}

\footnotetext{
* Supported by the U.S. Department of Energy, Basic Enerqy SciencesMaterials Sciences, under Contract W-31-109-Eng-38.

Manuscript submitted to the "Panel Study on Bonding and Adhesion at Solid State Interfaces," for the Council on Materials Science of the U.S. Department of Enerqy, Aspen, CO, August 11-16, 1985.
} 


\section{DISCLAIMER}

This report was prepared as an account of work sponsored by an agency of the United States Government. Neither the United States Government nor any agency Thereof, nor any of their employees, makes any warranty, express or implied, or assumes any legal liability or responsibility for the accuracy, completeness, or usefulness of any information, apparatus, product, or process disclosed, or represents that its use would not infringe privately owned rights. Reference herein to any specific commercial product, process, or service by trade name, trademark, manufacturer, or otherwise does not necessarily constitute or imply its endorsement, recommendation, or favoring by the United States Government or any agency thereof. The views and opinions of authors expressed herein do not necessarily state or reflect those of the United States Government or any agency thereof. 


\section{DISCLAIMER}

Portions of this document may be illegible in electronic image products. Images are produced from the best available original document. 
"Grain Boundaries in Ceramics and Ceramic-Metal Interfaces (D.R. Clarke, D. Wolf)

Three quite distinct interfaces are of interest, the crystal-crystal grain boundary found in certain very pure single phase ceramics, the crystal-glass-crystal grain boundary characteristic of the majority of single phase and polyphase ceramics, and the ceramic metal interface. Examples of the first class of boundary are to be found in ultra-high purity aluminas, magnesium oxide and a number of spinels. The second class of boundary is by far the most common, being present in almost all structural ceramics (aluminas, silicon nitrides, zirconias, etc.) and in the majority of electronic ceramics. The third class of boundary is also widespread and its importance is growing as greater interest (and applications) are devoted to ceramic-metal composites, metallization of ceramic substrates (including the multi-layer ceramic substrates) and metal particle dispersed ceramics.

For each of these interfaces the issues are similar, viz how are they held together, what is their structure, how can they be characterized and how and under what circumstances might they fail or de-adhere? The overriding need, however, is how these may be related so as to give an all-embracing picture of the adhesion (and conversely) failure of ceramic interfaces.

One significant difference between the grain boundaries in ceramics and the ceramic-metal interface is that the latter is usually subject to 
residual elastic stresses. This is clearly the case when metallization is deposited at high temperatures on a ceramic substrate having a different thermal expansion coefficient. Less obvious is the case of the formation of an oxide or corrosion scale on a metal component. Here, in addition to any thermal expansion mismatch stress, there are stresses resulting from the molar volume changes accompanying the formation of the oxide or scale.

\section{a) Bonding}

The detailed nature of the bonding at a ceramic grain boundary is poorly known at the present time, in part because of the lack of attention the subject has received until relatively recently. Some considerable insight has been gained through the use of computer simulations of grain boundaries in ionic ceramics (4) such as NiO and MgO, but to go substantially further will require a concerted approach using the full hierarchy of computer simulation techniques, starting with electronic structure calculations and progressing up to micro-mechanical simulations. This will require considerable effort but the results are thought to be especially worthwhile.

The bridging of the deep gap between electronic-structure calculations on one hand and central-force atomistic simulation methods on the other is particularly desirable and necessary. Electronic-structure calculations are capable of providing insight into the nature of the interactions between atoms in interfaces, the effect of impurities on these interactions, as well as the effect of a volume expansion near the interface. However, even in the distant future it appears impossible to apply these methods to the treatment of realistic interfaces directly due 
to the larger number of atoms involved. Hence, the insight gained at the electronic-structure level needs to be passed on to the atomistic level, for example, in the form of two- or three-body potentials. With the supercomputers available today, atomistic simulation techniques using interatomic potentials as input are capable of treating defects involving thousands of atoms. These techniques include lattice-statics, latticedynamics, Monte-Carlo, and molecular-dynamics methods. With the availability of good ab-initio interatomic potentials these techniques can be used to predict a variety of ideal-crystal, bicrystal, and point-defect properties of single metals, alloys, and predominantly ionic ceramic. materials. These properties include, for example, thermodynamic parameters such as the free energy of an interface, phase diagrams, melting and disordéring phenomena near interfaces, point-defect phenomena such as grain- or interphase boundary diffusion, and many others.

Whereas empirical central-force potentials have shown reasonable success in simple-metal and ionic-crystal grain-boundary calculations, their applicability to interphase boundaries between dissimilar materials (such as to metal-ceramic or metal-metal interphase boundaries) as well as to the effect of segregants on the interface structure and stability appears highly questionable and, indeed, unphysical in many cases. The establishment of the connection between electronic-structure and atomistic simulation methods would represent a major contribution towards overcoming the intrinsic limitations of both types of computer calculations. Without such an effort the role of computer simulation and theory must remain limited to relatively idealized special cases. 
The understanding of the structural and electonic properties of grain and interphase boundaries in predominantly ionic ceramic materials (such as metal oxides with $\mathrm{NaCl}$ structure, $\mathrm{Al}_{2} \mathrm{O}_{3}, \mathrm{ZnO}, \mathrm{TiO}_{2}$, and many others) could benefit substantially from greater efforts in the theoretical area. As is well known, the structural defect properties of these materials are intricately coupled with their electronic properties. This connection is due to the condition of overall charge neutrality of the bicrystal containing the grain or interphase boundary. In the past few years atomistic computersimulation methods have proven rather useful in predicting the structural defect properties and energetics of grain boundaries in such materials (4). On the other hand, the electronic properties of point defects in ideal crystals of alkali halides have been investigated by the color-center community for many decades (5). A combination of the theoretical methods developed for the understanding of the physics of color centers with what is known about point defects in grain boundaries in predominantly ionic ceramic materials would represent a major contribution toward the understanding and improvement of ceramic materials used in electronic devices (such as varistors, thyristors, sensors, capacitors, etc.).

On the experimental side, two approaches appear especially promising. One is the measurement of the electrical properties of synthetically produced bicrystals of known geometrical structure and well characterized chemistry. This would then provide the experimental benchmarks for the simulation and modelling experiments. The second approach is to try and extend the Tabor-Winterton-Israelachvili experiments (6-8) to measure the force directly between two ceramic surfaces. This is a formidable problem 
but one capable of providing the most direct measurement of the fundamental bonding forces (9).

The bonding of crystal-glass-crystal grain boundaries is an unresolved issue and is probably intimately connected with the question as to the stability of thin intergranular glass phases at the grain boundaries in ceramics. Given that it is energetically favorable for the grain boundaries to be wet by such intergranular phases at high temperatures, a continuum approach has been recently been proposed that gives the equilibrium thickness of the phase (10). The approach may be summarized by saying that the equilibrium thickness is dictated by the balance of two opposing forces, one the van der Wals dispersion force trying to bring the adjacent grains closer together, and the other being a repulsive force due to structural configurations of the liquid molecules trapped in between the grains. Although the approach may well be a useful starting point it begs the real question as to what the bonding across the interface really is in two respects. Firstly, it does not address the question of stability of the phase and secondly, as the predicted thickness is of the order of the molecular size of the liquid the continuum approach is perhaps not the most appropriate for obtaining quantitative predictions (11).

The bonding, and indeed structure, of the crystal-glass-crystal interface is one of widespread importance in materials science. At one extreme is the question of how amorphous materials bond to and arrange themselves at a crystal surface. At the other extreme is the question of how an amorphous or liquid phase (e.g. a lubricant) can support a normal stress without being squeezed out. (One of the unexpected conclusions of 
the continuum approach mentioned above is that a liquid phase at a ceramic grain boundary can indeed support a normal stress.) Another salient question, pertinent to polymer interfaces and to the Tabor-WintertonIsraelachvili experiment, is how molecules re-arrange themselves, and with what energy, when confined to volumes commensurate with their size. This is an interesting question in small system statistical mechanics. Some attention has been given to the problem recently including Monte-Carlo calculations (12) of the force on two absorbing plates separated by argon atoms.

b. Interfacial Structure

The emphasis of research activities to date in the ceramics field, and indeed much of materials science, has been placed on a geometrical description of the structure of grain boundaries (13). This is in line with the traditional approach that has been so successful in describing and modelling defect structures. However, with the increased recognition of dopants and additives and impurities in controlling such phenomena as grain growth, sintering, fracture toughness and now electronic properties of ceramics, it will become increasingly important to extend our description of interfaces to include their chemical and electronic characteristics.

\section{b.1 Geometric Structure.}

Investigations are al ready under way (Cornell, MIT, IBM and Stuttgart) in determining experimentally, principally by high resolution electron microscopy techniques, the atomic and defect structure of grain boundaries in pure, single phase, ceramics (14). This is essentially part of a 
larger, ongoing investigation seeking to relate interfacial geometric structure to the nature of the atomic bonding (viz metallic, ionic, covalent) across the interface. This has rapidly become conventional and is perhaps limited only by the man-power devoted to the investigations and the available resolution limits of the transmission electron microscope. Few unexpected results have so far been obtained, but this may well change with the advent of a new generation of microscopes having greatly improved resolutions in the next five years.

The logical extension of this work to the ceramic-metal interface is gaining momentum at the time of writing. Hitherto, this work has not been carried out, partly out of lack of tangible interest (this is changing because of the fresh impetus being given to ceramic-metal composites) and partly because of the difficulty in preparing suitable interfaces. The work of the Stuttgart group on the $\mathrm{Nb}-\mathrm{Al}_{2} \mathrm{O}_{3}$ interface (15) and the Philips group on the $\mathrm{Pt}-\mathrm{Al}_{2} \mathrm{O}_{3}$ interface (16) is especially noteworthy because of their choice of systems that exhibit particularly small thermal expansion mismatches; the large thermal stresses in other systems has always made it extremely difficult to prepare an integral sample.

In contrast, the structure of crystal-glass-crystal grain boundaries remains to be resolved, and it may well be that asking for a geometrical description is an inappropriate question until further progress is made in understanding what controls the thickness of intergranular phases in polyphase ceramics. According to recent ideas based on the existence of a structural disjoining pressure acting between grains with a glass phase separating them, the detailed arrangement of silicon tetrahedra between 
the grains and whether they have some form of epitaxial arrangement with respect to the crystal structure of the grain is of importance (10). However, it is the orientational ordering of the silica monomeric units that is important rather than the overall and complete geometry. The issue then becomes similar to a much wider generic question of interest in many branches of materials science (liquid crystals, polymer arrangements near surfaces, amorphous solids), that of the detailed structure of the interface between a crystalline and an amorphous phase. There are arguments, e.g. diffuse interface theory, that would suggest that, in contrast to a crystal-crystal interface, the interface is "smeared out" and has a "width" characterized by a correlation length.

\section{b.2. Electronic and Ionic Structure}

The electronic structure of grain boundaries in ceramics has been largely neglected since the works of Frenkel (17), Lehovac (18), and Kliewer and Koehler (19), who argued for the existence of a space charge region on either side of a grain boundary. This was extended recently by Yan et a1. (20) in the context of grain boundary diffusion and mobility in single phase, polycrystalline ceramics. The neglect of this topic is notable since there exists a great deal of empirically based knowledge on how to alter the resistivity of electronic ceramics by controlled heat treatments, atmospheres, and doping additions. It is also notable in view of the fact that a number of electronic ceramics, for instance the varistor materials and boundary layer capacitors, owe their exceptional properties exclusively to the electrical properties of their grain boundaries. 
The progress that has been made in the last few years comes from two principal sources, a) studies by the physics community on materials exhibiting similar varistor behavior (silicon bicrystals (21)) and b) complex impedance measurements on polycrystalline materials (22). Unfortunately, complementary characterization studies of the geometrical structure and chemical nature of the boundaries in the same materials have not been undertaken. As in other cases dealt with in this panel study, considerable progress is anticipated if carefully controlled bi-crystals (in this case $\mathrm{ZnO}$ ) were available.

\section{b.3. Chemical Structure}

Almost no ceramic material is pure and therefore characterization of the chemical structure of interfaces in ceramics is an imperative. Although the recent emphasis on chemically derived precursor materials (recommended by the Bowen panel (23)) in ceramic processing has raised the consciences of the ceramics community to the importance of impurities, they are invariably introduced during processing. (A common example is silica impurities originating from ball-milling.) Then, many ceramics cannot be fabricated without the use of additives to promote liquid phase sintering (e.g. silicon nitride ceramics) or additives to control grain growth or dopants to create a desired electrical property (e.g. Bi in Zno for varistor behavior).

That being said, lack of progress in this area is not for want of trying; many investigators have attempted to analyze the elements at ceramic interfaces, but with the exception of STEM/EDAX/EELS, few of the characterization tools used in related fields are applicable. Most 
ceramics, being insulators, charge up under electron and ion beams (the workhorses of modern surface analysis spectroscopies) making detailed identification difficult to impossible. Analytical electron microscopy has been used with considerable success for detecting elements segregating to grain boundaries, for analyzing the composition of inclusions and of large glassy regions. However, it lacks the spatial resolution required for analyzing their intergranular phases, whose thickneses are 6-20A. More seriously, however, analytical electron microscopy provides information only on the elements present, not on their chemical or bonding state. This is a particularly severe restriction since the necessity of charge balance is presumed to dictate that the chemical species can exist in a variety of ionic states.

c. Non-destructive Interface Characterization.

A real need exists for being able to characterize an interface that lies in the body of a material* without having to section or otherwise destroy the material, as one does in characterizing interfaces by TEM, Auger, SIMS etc. Development of such techniques would have widespread applicability throughout materials science.

Some significant, albeit small, progress toward this objective has been made in the last few years. At the highest spatial resolution are the attempts to use EXAFS to probe the atomic neighbors of solute atoms

\footnotetext{
*Variously known as a "buried" interface to the semiconductor community and an "internal" interface to the metallurgists.
} 
segregated to grain boundaries. Two such preliminary studies have been undertaken, Sn at grain boundaries in iron (24) and $\mathrm{Cu}$ at grain boundaries in $\mathrm{Nb}_{3} \mathrm{Sn}(25)$.

At slightly poorer resolution, are the techniques of glancing angle Xray diffraction $(26,27)$ now realizable with the use of synchrotron light sources. Although only a few initial experiments using these techniques have been performed, they will undoubtedly grow to become one of the mainstays in characterizing the roughness of interfaces and detect compound formation. It is possible that they will also find application for the detection of small area delaminations and flaws. The principal drawback of the glancing angle Xray techniques lies with the relatively small absorption length of the Xrays meaning that only interfaces within a few nundred to a few thousand angstroms can be probed. This will clearly restrict its use to ceramic-metal interfaces such as those of interest to the microelectronics field.

Related techniques, in which optical photons rather than Xray photons are used to probe the interface, are also expected to be more widely used. Of particular note are the guided wave spectroscopies in which an evavescent wave is used to probe the molecular structure of the interface. These techniques have been developed primarily for examining interfaces in polymeric systems $(28,29)$, both between one polymer and another and also between polymer molecules and inorganic substrates, but there is no reason why they cannot be fruitfully used for interfaces in ceramics. Indeed initial experiments are already underway for examining zirconia thin films. 
At the next level of resolution is the requirement to detect flaws and delaminations that might be responsible for subsequent adhesion failure. In this respect the problem is the same as that faced in non-destructive evaluation of flaws in structures and components. Considerable progress has been made in the development of techniques, protocols and the theory of scattering from defects and flaws, under the auspices of the DARPA initiated, and Air Force continuation, programs on NDE (30). The potential problem that needs to be addressed however is the nature of flaws responsible for adhesion failure; a necessary condition before the appropriate NDE techniques can be usefully applied. One feature of adhesion flaws that is expected to affect the type of NDE techniques is the fact that they are believed to lie within the interfacial region. Thus guided wave techniques in which the interrogation wave is propagated along the interface are likely to be especially informative.

One relatively new NDE technique that shows considerable promise for the examination of delaminations at interfaces is photo-acoustic spectroscopy (31).

\section{d. Fracture and Adhesion}

The mechanical failure of an interface is essentially a fracture phenomena, whether the interface is between two flat plates or grains, or between a sphere and a flat or between crossed-cylinders as used in the Tabor-Winterton-Israelachvili experiments. Invariably the stress required to separate an interface is much less than the theoretical bonding force of that interface, indicating that failure initiates and grows from a flaw or collection of flaws in the interface. 
A substantial body of fracture mechanics theory for the failure at an interface (including visco-elastic phenomena) has been developed in the last decade by Maugis et al. $(32,33)$ in France and Kenda! $1(34,35)$ in England with particular reference to adhesion. This has been substantially used to describe adhesion fracture of polymers in simple configurations, for instance for viscoelastic spheres on a rigid substrate, where the flaws are large (visible to the eye) or as in the peel test where the crack plane is very well defined. Whilst the theory is applicable to failure at ceramic and ceramic-metal interfaces, the problem has been to identify the flaw responsible for de-adhesion. For this reason, there is a growing recognition, as in the fracture mechanics of brittle materials and structural ceramics, that detailed study must make use of deliberately introduced, controlled sized flaws (36). Thus, work is beginning to measure the fracture toughness of individual grain boundaries and of individual ceramicmetal interfaces by propagating fracture from an indentation produced crack. Obviously this requires the capability to form bicrystals and single interfaces between a metal and ceramic. Such investigations have the clear potential of relating, for the first time, the fracture toughness of an interface to its structure.

The situation of thin metal films on a ceramic (or otherwise brittle) substrate is less advanced, primarily because of the lack of a fracture mechanics framework to describe crack growth below a thin film (i.e. the fact that the thin film is not a semi-infinite continuum). However, two recent analyses $(37,38)$ on the delamination of thin films possibly point the way for future work. Both are based on the formation of a flaw by a buckling phenomenon in the thin film driven by the residual stress in the 
film (resulting from, for instance, thermal expansion mismatch). How one may introduce a controlled flaw also remains an unresolved issue.

As the driving force for de-adhesion is usually the development of elastic stress, there is a need for methods of monitoring stress as an interface is formed and of measuring stress (and, in particular, stress gradients) after the interface has been created. This is especially so for metal-ceramic interfaces. One traditional approach has been to measure the curvature of an elastic substrate, say a silicon wafer, during and after deposition of a film. Another standard method is to use Xray diffraction techniques to measure the inter-atomic spacing and thereby deduce the stress. Both of these are averaging techniques in the sense that the average stress over all the film is measured. New possibilities for in-situ and remote measurement of stress from point to point are offered by Raman micropobe spectroscopy $(39,40)$. To date the use of this technique has been restricted to stress measurement in silicon wafers in the vicinity of metallization and laser melted tracks $(41,42)$, but there is no technical reason why it cannot be applied to a much wider range of ceramic materials. The basis of this technique is that elastic strains alter the vibrational frequencies of lattice phonons and thereby shift the positions of the peaks in the Raman spectra. As the laser beam used in Raman microprobe spectroscopy can be focused down (according to the optics employed) to regions as small as 1 um square, it is possible to measure stresses with this sort of spatial resolution. Advances in high power lasers and parallel detection of wavelength dispersed spectra promise to increase the speed at which the stresses may be measured. 
In-situ characterization of elastic stresses remains a formidable challenge before detailed modelling and comparison with experiment can be undertaken. It is also a challenge shared with many other interfaces.

Fracture in polycrystalline and polyphase ceramics is commonly intergranular, yet little is known to date on what determines whether the fracture is inter- or intragranular. Likewise, we have little predictive capabilities on ascertaining which boundaries will fail. It is presumed that intergranular fracture occurs because the boundaries are weaker than the grains but why is not known. There are suggestions that segregation of impurities, i.e. calcium to grain boundaries in alumina, lower the fracture tojghness of the material. To demonstrate this, well defined bi-crystal experiments in which impurity segregation can be controlled are sorely needed. As the overall fracture toughness of a ceramic is some sort of weighted average of the fracture toughness of the individual boundaries, a statistical approach is required and so it is expected that computer simulation of crack propagation through a polycrystalline material will become a fruitful avenue of research.

Of particular interest is the time dependent fracture of a crystalgiass-crystal interface at high temperatures, pertinent to high temperature structural applications of ceramics. This is known to be an exceedingly complex mode of failure, involving viscous flow of the glass, cavitation within the glass, the possibility of solution-reprecipitation, grain boundary sliding and migration. This is compounded by many of these processes being deformation rate and temperature dependent. 


\section{RECOMMENDATIONS}

A number of points made in the above text are emphasized below as specific recommendations for future exploration.

1. Use of Ceramic Bicrystals. The availability of bicrystals will enable well-defined interfaces to be studied and eventually their fracture properties be correlated to such variables as structure, orientation, impurity concentration, etc. They will also be especially useful for exploring how electronic properties might be related to the nature of the boundaries. The major problem at the present time is the lack of such bicrystals and the difficulty in manufacturing them. DOE could take a lead role in fostering the development of facilities for the preparation of such bicrystals. Both ceramic-ceramic bicrystals and crystal-glass-crystal bicrystals would be required.

2. Wetting Studies of Grain Boundaries. Although wetting studies have been out of fashion for over a decade, a new impetus on the wetting of grain boundaries is required for two reasons. One is a scientific reason; as a way to obtain the energy of a grain boundary as a function of its structure for comparison with the predictions of theoretical models. (It is noted that whilst the detailed atomic structure of an interface depends sensitively on the choice of interatomic potential the calculated energy of the interface does not). The second reason is to ascertain experimentaliy under what conditions of composition and temperature a liquid phase will wet or de-wet a ceramic grain boundary. Such empirical information would 
provide a valuable data base for, for instance, designing liquid phase sintering systems and the development of boundary layer capacitors.

3. Increased Support for Computational Simulation Activities. The implementation of an interdisciplinary theoretical effort similar to that outlined here in which a hierarchy of computational models, bridging electronic structure calculations to atomistic and micromechanical simulations, is developed will require the availability of substantial amounts of supercomputer time. The needs exceed the present, rather limited, availability of such supercomputer access to DOE-BES funced research groups.

4. Dévelopment of Methods for the Non-destructive Examination of Interfaces. There is a real need for techniques to examine interfaces in-situ and non-destructively. Few, if any, such techniques exist at the present time. Whilst it is unlikely that any remote technique will rival the spatial resolution and analytical capabilities of the analytical electron microscope and associated surface analys is techniques, the capability of characterizing an interface directly prior to testing its mechanical integrity or fracture toughness will enable direct correlations to be developed. Of the more promising techniques proposed, mention might be made of Raman microprobe spectroscopy, glancing angle Xray diffraction, wave-guide mode spectroscopies and photo-acoustic spectroscopy. 
5. Adhesion measurements using controlled flaws. It is recognized that much of the variability in adhesion measurements can be attributed to the variability in flaw size and lack of knowledge of those flaws. To overcome this limitation it is recommended that deliberately introduced flaws be used, much as indentation induced flaws have revolutionized our understanding of brittle fracture and strength in bulk structural ceramics. 


\section{REFERENCES}

1. "Characterization of Grain Boundaries", Advances in Ceramics Vol. 6, edited M.F. Yan and A.H. Heuer, American Ceramic Society 1983.

2. "Structure and Properties of Internal Interfaces" edited M. Ruhle et al. Journal de Physique, C4, 1985.

3. "Grain Boundaries In Semiconductors", edited H.J. Leamy, G.E. Pike and C.H. Seager, North Holland, 1982.

4. See, for instance, articles in reference 2, and

4a. P.W. Tasker and D.M. Duffy, "Computer Simulation of (001) Tilt Grain Boundaries in Nickel 0xide", Philos. Mag. 47817 (1983).

4b. D. Wolf, "Energy and Structure of (001) Coincident Site Twist Boundaries and the Free (001) Surface in Mgo: A Theoretical Study", Journal of the American Ceramic Society, 67 (1984).

4C. D.M. Duffy and P.W. Tasker, "Space Charge Regions Around Dipolar Grain Boundaries", 56971 (1984).

4d. A.B. Lidiard and M.J. Norgett, in "Computational Solid State Physics" edited F. Herman, N.W. Dalton and T.R. Koehler, Plenum Press, New York, 1972.

4e. M.J. Norgett, "A General Formulation of the Problem of Calculating the Energies of Lattice Defects in Ionic Crystals", AERE Report R7650 Harwe11, 1974.

5. A.M. Stoneham, "Theory of Defects in Solids: Flectronic Structure of Defects in Insulators and Semiconductors", Uxford University Press, 1975.

6. D. Tabor and R.H.S. Winterton, "The Direct Measurement of Normal and Retarded van der Waals Forces," Proc. Roy. Soc. A312 435-450 (1969).

7. J.N. Israelachvili and D. Tabor, "Measurement of van der Waals Dispersion Forces in the Range 1.5 to $130 \mathrm{~nm}^{\prime \prime}$, Proc. Roy. Soc. A331 19 (1972).

8. J.N. Israelachvili and G.E. Adams, "Measurement of Forces Between Two Mica Surfaces in Aqueous Electrolyte Solutions in the Range $0-100 \mathrm{~nm}^{\prime}$, J. Chem. Soc., Faraday Trans. I. 74975 (1978).

9. J.N. Israelachvili, "Intermolecular and Surface Forces", Academic Press, Orlando (1985).

10. D.R. Clarke, "On the Equilibrium Thickness of Intergranular Glass Phases in Ceramic Materials", J. Amer. Ceram. Soc., In Press.

11. R.G. Horn and J.N. Israelachvili, "Direct Measurement of Forces Due to Solvent Structure", Chemical Phys. Lett. 71192 (1980). 
12a. J.E. Lane and T.H. Spurling, "Forces Between Adsorbing Walls: Monte Carlo Calculations", Chemical Physics Letters 67107 (1979).

12b. J.E. Lane and T.H. Spurling, "Monte Carlo Simulation of the Effects of Adsorption on Interparticle Forces", Australian J. Chem. 33231 (1980).

13a. A.P. Sutton, "Grain Boundary Structure", International Metallurgical Review, 29377 (1984).

13b. R.C. Pond and D.S. Vlachavas, "Bi-crystallography", Proc. Roy. SoC. A386 95 (1983).

13c. R.C. Pond, D.A. Smith and V. Vitek, "A Model for Grain Boundary Structure Based on Random Close Packing", Scripta. Met. 12699 (1978).

14. See, for instance, article in references 1-3, and

14a. P. Chaudhari and J.W. Matthews, "Coincidence Twist Boundaries Between Crystalline Smoke Particles", Journal of Applied Physics, 423063 (1971).

14b. R.W. Balluffi, P.D. Bristowe and C.P. Sun, "Structure of High Angle Grain Boundaries in Metals and Ceramic Oxides", Journal of the American Ceramic Society, 6423 (1981).

14C. C.B. Carter, Z. El gat and T.M. Shaw, "First Order Twin Boundaries in Spinel: The Incoherent Twin", Philos. Mag., In Press.

15. M. Florjancic, W. Mader, M. Ruhle and M. Turwitt, "HREM and Diffraction Studies of $\mathrm{Al}_{2} \mathrm{O}_{3} / \mathrm{Nb}$ Interfaces", in reference 2.

16. J.T. Klomp, "Ceramic-Metal Interactions" in "Electronic Packaging Materials Science", edited K.N. Tu and E. Giess, Materials Research Society, 1985.

17. J. Frenkel, "Theory of Liquids", Oxford University Press, 1946.

18. K. Lehovac, "Space-charge Layer and Distribution of Lattice Defects at the Surface of Ionic Crystals", Journal of Chemical Physics 211123 (1953).

19. K.L. Kliewer and J.S. Koehler, "Space Charge in Ionic Crystals: I", Phys. Rev. 1401226 (1965).

20. M.F. Yan, R.M. Cannon and H.K. Bowen, "Space Charge, Elastic Field and Dipole Contributions to Equilibrium Solute Segregation at Interfaces", Journal of Applied Physics 54764 (1983).

21. See for instance, C.H. Seager and G.E. Pike, "Grain Boundary States and Varistor Behavior in Silicon Bicrystals", Applied Physics Letters, 35709 (1979). 
22. See, for instance, E.P. Butler and N. Bonanos, "The Characterization of Zirconia Engineering Ceramics By A.C. Impedance Spectroscopy", Materials Science and Engineering, 7149 (1985).

23. "High Temperature Ceramics". A report from the Council on Materials Science chaired by H.K. Bowen, January 1979. Materials Science and Engineering 441 (1980).

24. W.J. Pardee, W.M. Robertson and M.R. James, "Tin Segregation at Iron Grain Boundaries Measured by EXAFS", Scripta Met. 141333 (1980).

25. M. Suenaga, Private Communication.

26. W.C. Marra, P. Eisenberger and A.Y. Cho, Journal of Applied Physics 50 $6927(1979)$.

27. S.M. Heald, J.M. Tranguada, H. Chen and D.0. Welch, "Grazing Incidence Xray Methods for the Characterization of Coatings and Coating/Substrate Interfaces", Applied Physics Letters, In Press.

28. J.F. Rabolt, N.E. Schlotter and J.D. Swalen, "Spectroscopic Studies of This Film Polymer Laminates Using Raman Spectroscopy and Integrated Optics", J. Physical Chem. $854141,1981$.

29. J.F. Rabolt, R. Santo and J.D. Swalen, "Raman Spectroscopy of Thin Polymer Films Using Integrated Optical Techniques", Appl. Spectrosc. $33549,1979$.

30. "Review of Progress in Quantitative Non-Destructive Evaluation", Vol I \& II, ed. by D.O. Thompson and D.E. Chinenti, Plenem Press (1981 and 1983).

31. A. Rosencwaig, "Photoacoustics and Photoacoustic Spectroscopy", J. Wiley, New York 1980.

32. D. Maugis and M. Barquins, "Fracture Mechanics and Adherence of Viscoelastic Bodies", J. Phys. D. Appl. Phys. 111989 (1978).

33. D. Maugis, "Overview of Adherence Phenomena", in Adhesive Chemistry ed. Leing-Huang Lee, Plenum, 1984.

34. K. Kenda11, "The Adhesion and Surface Energy of Elastic Solids", J. Phys. D. Appl. Phys. 41186 (1971).

35. K. Kendall, "Thin Film Peeling - The Elastic Term", J. Phys. D. Appl. Phys. 1449 (1975).

36. B.R. Lawn, "The Indentation Crack As A Model Surface Flaw", in "Fracture Mechanics of Ceramics" vol. 5, edited R.C. Bradt, A.G. Evans, D.P.H. Hasselman and F.F. Lange, Plenum, 1983.

37. A.G. Evans, and J.W. Hutchinson, "On the Mechanics of Delamination and Spalling In Compressed Films", Intern. J. Solids Structures 20 (1984). 
38. D.J. Srolovitz and M.P. Anderson, "A Criterion for Compressive Failure of a Continuous, Protective Surface Film", Acta Met. 321089 (1984).

39. M. Delhaye and P. Dhamelincourt, "Raman Microprobe and Microscope With Laser Excitation", J. Raman Spectrosc. 333 (1975).

40. F. Adar and D.R. Clarke, "Raman Microprobe Spectroscopy of Ceramics", Microbeam Analysis - 1982, ed. K.F.J. Heinrich, San Francisco Press, 1982.

41. S.A. Lyon, R.J. Nemanich, N.M. Johnson and D.K. Biegelsen, "Microstrain in Laser Crystallized Silicon Islands on Fused Silica", Applied Phys. Lett. 40316 (1982).

42. P. Zorabedian and F. Adar, "Measurement of Local Stress in Laser Recrystallized Lateral Epitaxial Silicon Films Over Silicon Dioxide Using Raman Scattering", Appl. Phys. Lett. 43177 (1983). 